\title{
A high-resolution optically addressed spatial light modulator based on $\mathrm{ZnO}$ nanoparticles
}

\author{
Pawan Kumar Shrestha, Young Tea Chun and Daping Chu
}

An optically addressed spatial light modulator (OASLM) can modulate the wavefront of a read light by displaying a phase pattern or a hologram configured by the intensity distribution of a write light. Using ZnO nanoparticles (NPs) as a novel photoconductor, a high-resolution OASLM was fabricated. A ZnO NP suspension was spin-coated on an indium tin oxide (ITO)-coated glass substrate and annealed to form a photosensitive layer. The device was characterized electrically and optically. The device was operated at low driving voltages in the transmission mode. Updatable recording of a diffraction grating up to $825 \mathrm{Ip} \mathrm{mm}^{-1}$ with a diffraction efficiency (DE) of $0.05 \%$ and binary holograms with pixel sizes from $2 \mu \mathrm{m}$ down to $0.72 \mu \mathrm{m}$ were demonstrated using a $405 \mathrm{~nm}$ wavelength write laser and a $635 \mathrm{~nm}$ wavelength read laser.

Light: Science \& Applications (2015) 4, e259; doi:10.1038/lsa.2015.32; published online 13 March 2015

Keywords: holography; nanoparticles; OASLM; ZnO

\section{INTRODUCTION}

A hologram as a recorded interference pattern consists of both amplitude and phase information of the light diffracted from the target object. When the read light passes through such an interference pattern, it recreates the replay image of the recorded object, which can give viewers a sensation of viewing a real three-dimensional (3D) object. To provide a wide viewing angle, the pixel pitch of the recording media needs to be comparable to or smaller than the wavelength of light. This is difficult to achieve using the current electrically addressable spatial light modulators (EASLMs), such as phase-only liquid crystal on silicon devices or digital mirror devices. In contrast, the structure of an optically addressed spatial light modulator (OASLM) enables very high resolution without the need for complicated fabrication processes. Moreover, for pixelated EASLMs, dead space between pixels is inevitable, which acts as a two-dimensional diffraction grating, resulting in an undesirable diffraction pattern on top of the desired image. However, OASLMs have no physically pixilated electrodes, and they do not require complicated electronic addressing circuitry to drive. The absence of inter-pixel gaps and ease of fabrication and operation together with the extremely high resolution achievable for written phase patterns make the OASLM a favorable choice for holographic displays.

It is desirable to have an OASLM that is capable of forming highresolution holograms over a large active area and that can be easily fabricated at a low cost. The resolution of OASLMs is largely limited by lateral charge diffusion in the photoconductor layer and the fringing electric field profile in the liquid crystal (LC) layer. Traditionally, a$\mathrm{Si}: \mathrm{H}$ materials were used as photosensors in photoconductor or photodiode configurations. ${ }^{1-7}$ Fukushima et al. ${ }^{1,2}$ demonstrated an OASLM with a resolution of $175 \mathrm{lp} \mathrm{mm}^{-1}$ and a diffraction efficiency
(DE) of approximately $0.01 \%$ using an a-Si:H film. This was a significant improvement over the previously reported resolution of $\sim 50 \mathrm{lp}$ $\mathrm{mm}^{-1}$. With the help of a C-doped a-Si:H film, the OASLM resolution was further improved to $370 \mathrm{lp} \mathrm{mm}{ }^{-1}$ with a peak DE of $8 \% .^{8}$ In this case, the modulation transfer function at $50 \%$, generally considered an indication of the spatial resolution of the device, was measured to be

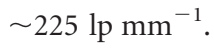

Other materials used as photosensors in LC OASLMs include $\mathrm{ZnO},{ }^{9}$ BSO,${ }^{10}$ crystalline silicon, ${ }^{3,4} \mathrm{As}-\mathrm{Se},{ }^{5} \mathrm{a}-\mathrm{As}_{2} \mathrm{~S}_{3}{ }^{6}$ and phthalocyanine. ${ }^{7}$ In parallel, photorefractive polymers exhibited the ability to record interference fringe patterns of micron sizes, although very high voltages of 5-10 kV must be applied during operation for a device 50-100 $\mu \mathrm{m}$ thick. ${ }^{11}$ Using certain combinations of photoconducting polymers and LC materials, high-resolution devices have been achieved with relatively low driving voltages. ${ }^{12}$ An interference period of $1.9 \mu \mathrm{m}$ $\left(527 \mathrm{lp} \mathrm{mm}{ }^{-1}\right.$ ) with a DE of $1.5 \%$ was reported when using a nematic LC (NLC) with $\mathrm{C}_{60}$-doped poly( $\mathrm{N}$-vinylcarbazole) at an operating voltage of $80 \mathrm{~V}$ direct current (DC). ${ }^{13}$

A typical OASLM device consists of a photosensitive film next to a layer of a light-modulating material, with both of these layers sandwiched between two pieces of indium tin oxide (ITO)-coated glass. The resolution of an OASLM is dependent on the lateral diffusion length of charge carriers in transit. Wang and Moddel ${ }^{14}$ developed a transient charge transport model to simulate the limits of resolution of an OASLM. The write light, incident on the photosensor layer, generates the charge carriers. These charge carriers diffuse and drift until they are trapped or the write light is off. Introducing defects at the interface to trap charge carriers can improve the resolution. In the case of OASLMs with crystalline semiconductors, pixelating the interface can impede the charge spreading effect. ${ }^{14,15} \mathrm{~A}$ thin photoconductor 
layer helps minimize lateral charge spread, but it may be difficult to fabricate a thin crystalline photoconductor, or the sensitivity of the thin layer may be low.

In this work, we use a $\mathrm{ZnO}$ nanocolloidal suspension in ethanol to form a novel nanoparticle (NP)-based photosensor for an OASLM. $\mathrm{ZnO}$ NPs can exhibit a photo-current on/off ratio of six orders of magnitude. The high absorption of UV light by the ZnO NP layer enables the fabrication of a very thin photosensor layer. The $\mathrm{ZnO}$ NP layer has a high defect density and hence lower charge mobility compared to a crystalline $\mathrm{ZnO}$ layer. These properties suggest the potential to achieve high spatial resolutions for updatable holograms using such a ZnO NP layer. The proposed OASLM device consists of a $\mathrm{ZnO}$ NP photoconductor film next to a layer of NLC material, with both of them in between two pieces of ITO-coated glass, as shown in Figure 1a. We succeed in recording a fringe period as small as $1.2 \mu \mathrm{m}$ (825 $\mathrm{lp} \mathrm{mm}^{-1}$ ) with a DE of $0.05 \%$.

Solution-processed $\mathrm{ZnO}$ NPs offer several advantages in terms of flexible deposition, easy customization of material properties, low cost fabrication and the ability to be applied on large-area devices. ${ }^{16,17} \mathrm{ZnO}$ NPs can be spin-coated on top of different types of substrates and annealed in a reasonably low temperature range of $100-400{ }^{\circ} \mathrm{C}$ to form a $\mathrm{ZnO} \mathrm{NP}$ photoconductor film. Unlike nanowires, NPs require no alignment to enhance device performance. These features of $\mathrm{ZnO}$ NPs will allow future applications in flexible OASLMs. Furthermore, $\mathrm{ZnO}$ NP photosensor films can be used together with bi-stable ferroelectric LC materials to enable active tiling of holograms on OASLMs for large
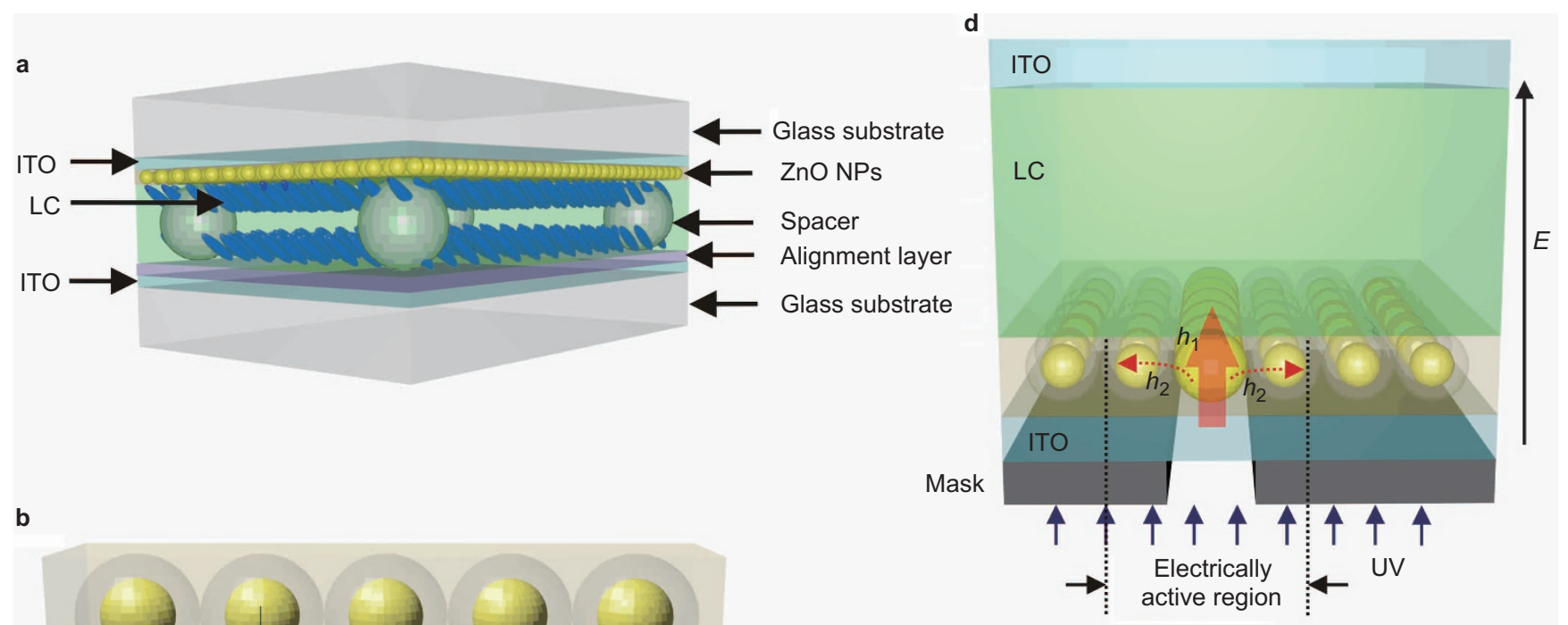

b
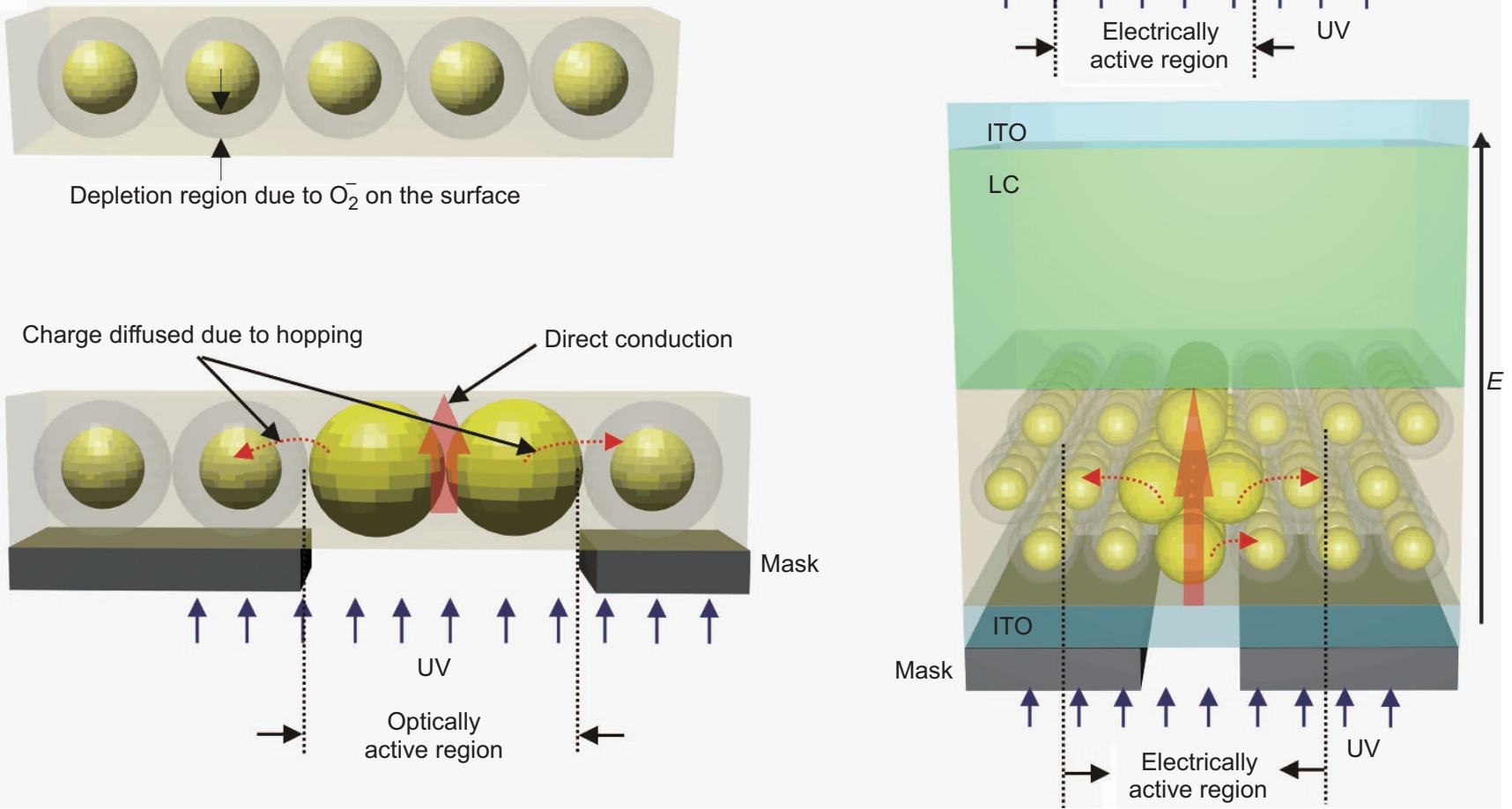

c

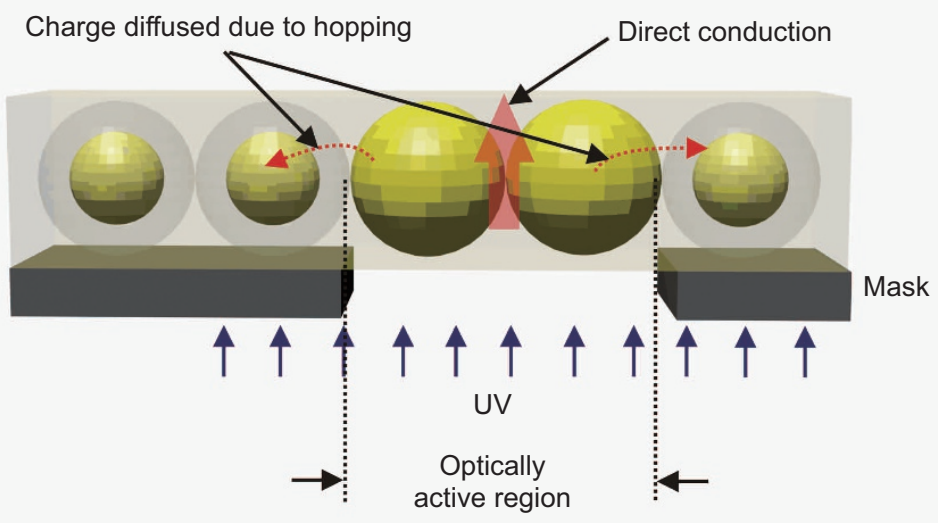

Figure 1 Device structure and hypothesized carrier transport. (a) OASLM device structure. (b) ZnO NP layer showing depletion region. (c) Disappearance of depletion region under UV illumination. (d) Charge hopping for a single and a multi-layer of ZnO NPs under UV illumination and applied voltage. For a single layer, $p_{h} \sim p_{h 0} \exp \left(-d / d_{0}\right)$, and for a multi-layer, $\sigma_{\|} / \sigma_{\perp} \sim p_{h 2} / p_{h 1} \sim \exp \left(-d_{\mathrm{d}} / d_{0}\right)$ and $\sigma_{\|} \sim \sigma_{\perp}$. Here $p_{h}$ is the probability of a charge carrier hopping a distance $d$, $d_{d}$ is the depletion distance, $d_{0}$ is the mean free path, $p_{h 0}$ is the normalized hopping probability within the mean free path, $p_{h 1}$ and $p_{h 2}$ are the probabilities of hopping through paths $h_{1}$ and $h_{2}$, respectively, and $\sigma_{\|}$and $\sigma_{\perp}$ are the lateral and orthogonal conductivities, respectively. ITO, indium tin oxide; LC, liquid crystal; NP, nanoparticle; OASLM, optically addressed spatial light modulator; UV, ultraviolet. 
updatable holographic displays. ${ }^{18} \mathrm{ZnO}$ itself has a wide band gap $\left(E_{\mathrm{g}}=3.4 \mathrm{eV}\right)$ and is transparent to visible light. Undoped $\mathrm{ZnO}$ is normally $n$-type because of oxygen vacancies in the material. ${ }^{19-21}$ Oxygen atoms on the surface of a $\mathrm{ZnO} \mathrm{NP}$ adsorb free electrons through a process of $\mathrm{O}_{2}(\mathrm{~g})+\mathrm{e}^{-} \rightarrow \mathrm{O}_{2}{ }^{-}$, resulting in a depletion region of low dark conductivity near its surface. When the $\mathrm{ZnO}$ NP film is subjected to suitable UV light radiation, electron-hole pairs are generated. The generated holes can recombine with the electrons adsorbed by the oxygen atoms at the $\mathrm{ZnO} \mathrm{NP}$ surfaces through a process of $\mathrm{h}^{+}+\mathrm{O}_{2}{ }^{-}(\mathrm{ad}) \rightarrow \mathrm{O}_{2}(\mathrm{~g})$. This leads to an increase in the free electron density and a decrease in the thickness of the depletion region and hence increases the conductivity of the $\mathrm{ZnO} N P$ film. ${ }^{20,22-24}$ When the UV radiation is turned off, the oxygen atoms re-adsorb the free electrons again, decreasing the conductivity of the photoconductor film. The large surface-to-volume ratio of the $\mathrm{ZnO}$ NPs results in a large depletion region being formed on the NP surfaces in the dark state. This makes the conductivity of the ZnO NPs very sensitive to UV radiation, allowing the use of a relatively thin photosensor layer in an OASLM. The conduction mechanism of a NP film in this case is significantly different from that of a bulk single crystal or an amorphous film, where the injected electrons hop from particle to particle. ${ }^{25}$ During the OASLM operation, we have an optical region of illumination. This region has an increased number of charge carriers and hence an increased conductivity, making it an electrically active region. However, the size of the whole electrically active region for the LC switching is determined by the charge diffusion coefficient and the illumination time (Figure $1 \mathrm{~b}$ and 1c). The illumination time has to be greater than the LC switching time (for an NLC, $\sim 10 \mathrm{~ms}$, and for a ferroelectric LC, $\sim 10 \mu \mathrm{s}$ ), and the charge diffusion coefficient of the NP ZnO film is $1.1 \times 10^{-4} \mathrm{~cm}^{2} \mathrm{~s}^{-1}$, which is much smaller than that of a bulk film due to the NP's surface depletion region and the single particle stacking layer of the film. ${ }^{26}$ Hence, we can envisage that a monolayer $\mathrm{ZnO}$ NP film would have a greater vertical charge carrier mobility and a lower lateral diffusivity due to the hopping conduction mechanism illustrated in Figure 1d, thus increasing the resolution of the OASLM. $^{14}$

The resolution of an OASLM is one of the fundamental factors limiting the viewing angle (Figure $2 \mathrm{a}$ ) and is given by
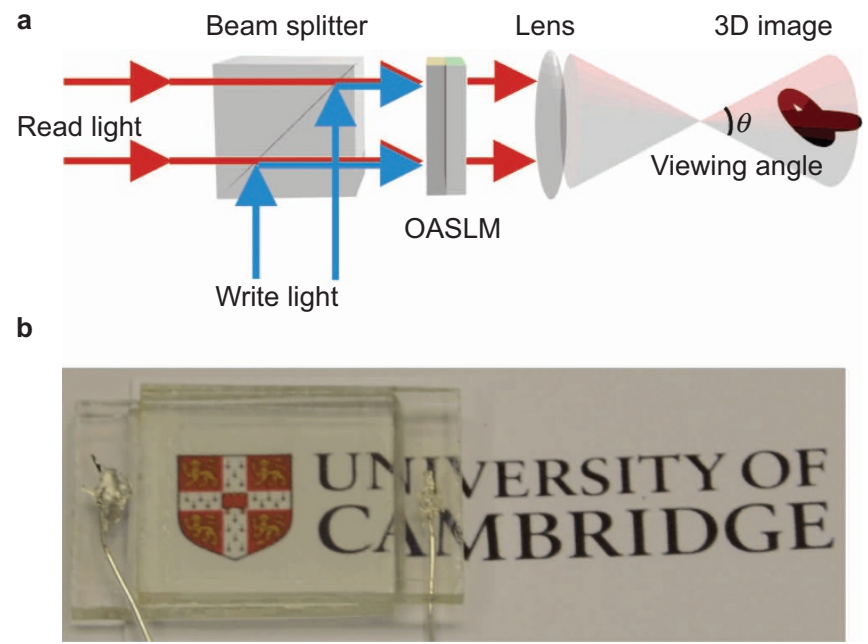

Figure 2 (a) An illustration of the viewing angle of a 3D image on an OASLM. (b) An image of a fabricated ZnO NP OASLM cell in which the logo of the University of Cambridge underneath the cell is clearly visible. 3D, three-dimensional; NP, nanoparticle; OASLM, optically addressed spatial light modulator.

$$
\theta= \pm \sin ^{-1} \frac{\lambda}{2 \times \text { pixel pitch }}
$$

where $\theta$ is the viewing angle and $\lambda$ is the wavelength of light.

\section{MATERIALS AND METHODS}

\section{Device fabrication}

A typical $\mathrm{ZnO}$ NP OASLM fabricated in our lab is shown in Figure 2b. ITO-coated glass substrates $16 \mathrm{~mm} \times 21 \mathrm{~mm}$ in size $\left(\sim 30 \Omega \mathrm{sq}^{-1}\right)$ were cleaned thoroughly by Acetone/IPA sonication. A ZnO NP suspension in ethanol (Sigma Aldrich Co., Gillingham, Dorset, UK) was spin-coated on top of ITO-coated glass followed by annealing at $180{ }^{\circ} \mathrm{C}$ for $10 \mathrm{~min}$. The $\mathrm{ZnO}$ NP suspension we use contains NPs with an average size of approximately $35 \mathrm{~nm}$ and a maximum size $<130 \mathrm{~nm}$. Therefore, we chose to prepare an $80 \mathrm{~nm}$ thick ZnO NP film. The layer thickness was measured using Dektak. Figure 3 a shows the atomic force microscope image of the deposited NP layer, and the RMS roughness measured in a $1 \mu \mathrm{m}^{2}$ area is $4.65 \mathrm{~nm}$. The photosensor layer was rubbed for LC alignment. Polyimide (AM4276) was spincoated on a separate ITO-coated glass substrate and baked at $140{ }^{\circ} \mathrm{C}$ for $5 \mathrm{~h}$ followed by rubbing. The cell was fabricated by gluing these two rubbed layers in an anti-parallel configuration with the alignment directions facing each other and $5.5 \mu \mathrm{m}$ spacers between them. The cell gap was measured to be $5.7 \mu \mathrm{m}$ using a Fizeau interferometer. The cell was then filled with NLC E7 (Merck, Hull, UK) via the capillary effect. The filling hole of the cell was sealed with glue, and the electrical connections were soldered. Reproducibility of the process was confirmed by fabricating five working OASLMs. These devices have been stored at normal room temperature for more than eight months, and no degradation has been observed.
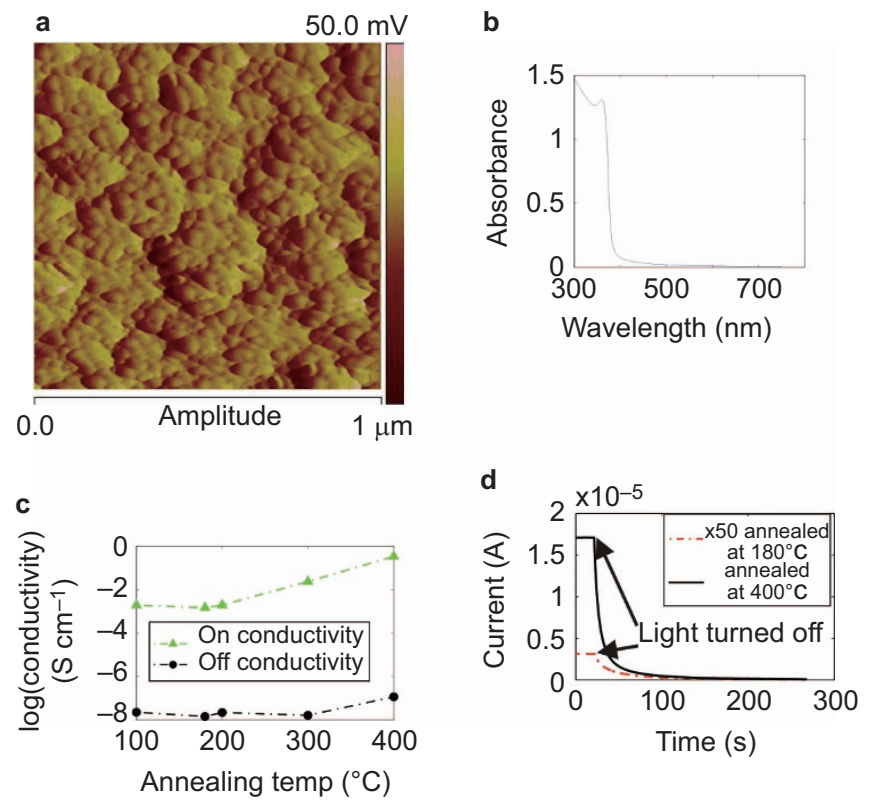

Figure 3 (a) Atomic force microscope image of the ZnO NP layer. (b) UV-vis absorption spectra of the ZnO NP layer. (c) Relation of ZnO NP on/off conductivity of films annealed at various temperatures. (d) Temporal current response with and without illumination. Samples are illuminated by $10 \mathrm{~mW} \mathrm{~cm}{ }^{-2}$ from a $365 \mathrm{~nm}$ light emitting diode with 6 V DC applied throughout. DC, direct current; NP, nanoparticle; UV-vis, ultraviolet-visible. 


\section{Electrical characterization}

For conductivity measurements, two rectangular-patterned ITO electrodes, each $18 \mathrm{~mm}$ in length and $4.5 \mathrm{~mm}$ in width with a separation of $500 \mu \mathrm{m}$, on glass were used. Eighty-nanometer thick ZnO NP layers were deposited by spin-coating and then annealed at different temperatures. $I-V$ measurements of the corresponding dark/photo currents of the ITO-ZnO-ITO structures were carried out using an Agilent 4156 (Yokogawa-Hewlett-Packard Ltd, Hachioji-Shi, Tokyo, Japan) together with a $365 \mathrm{~nm}$ light-emitting diode light source of $2 \mathrm{~mW} \mathrm{~cm}{ }^{-2}$. The conductivity $\sigma$ of the sample is calculated as

$$
\sigma=\frac{d I}{L t V}
$$

where $d$ is the separation of the electrodes, $I$ is the measured current, $L$ is the length of the electrodes, $t$ is the thickness of the layer and $V$ is the applied voltage. The $I-V$ measurements show that ohmic contact is formed at the $\mathrm{ZnO}-\mathrm{ITO}$ interface. For an annealing temperature of $180{ }^{\circ} \mathrm{C}$, photo/dark (on/off) conductivities were measured to be $0.001 \mathrm{~S} \mathrm{~cm}^{-1}$ and $1.44 \times 10^{-8} \mathrm{~S} \mathrm{~cm}^{-1}$, respectively. The spectrometry results in Figure $3 \mathrm{~b}$ show that the absorbance of our $\mathrm{ZnO}$ NP film peaks at $360 \mathrm{~nm}$ and rapidly falls as the wavelength increases. This visibly transparent material shows a sharp decrease in the photocurrent in the range of 400 to $700 \mathrm{~nm} .{ }^{23}$ When excited by a laser ( $405 \mathrm{~nm}, 18 \mathrm{~mW} \mathrm{~cm}^{-2}$ ), the photoconductivity is measured to be $2.64 \times 10^{-7} \mathrm{~S} \mathrm{~cm}^{-1}$, which is low compared to the light emitting diode excitation. Nevertheless, the prepared photoconductor film is sufficiently sensitive to modulate the LC materials in an OASLM. Our preliminary experiment shows that at $473 \mathrm{~nm}$, a powerful $5 \mathrm{~mW}$ laser is needed as a write light. A detailed investigation is necessary to determine the quality of the image produced. A decrease in the NP size leads to a shift of the peak absorption wavelength toward shorter wavelengths due to the quantum confinement effect. It has been demonstrated that the peak absorption wavelength shifts from $360 \mathrm{~nm}$ to $310 \mathrm{~nm}$ as the particle size decreases. ${ }^{27,28}$ This suggests that our OASLM with a smaller NP size may be able to operate with a UV light as the write light and red, green and/or blue light as the read light.

As shown in Figure 3c, the on/off current ratio increases with the annealing temperature in the range between 100 and $400{ }^{\circ} \mathrm{C}$. Low dark current is very important for producing high quality images. ${ }^{10}$ Unfortunately, for the film annealed at a higher temperature, the current remains high for a longer period after the illumination is turned off relative to that for a film annealed at a lower temperature (Figure 3d). The minimum annealing temperature has to be higher than the boiling point of the solvent used for the suspension of NPs. OASLMs were fabricated with $\mathrm{ZnO}$ NP layers annealed at temperatures between 100 and $180{ }^{\circ} \mathrm{C}$, and they showed very similar electricaloptical performances. In this work, we chose to use $180{ }^{\circ} \mathrm{C}$ annealing to ensure satisfactory solvent drying.

\section{RESULTS AND DISCUSSION}

Optical testing of the fabricated OASLM was carried out in transmission mode using the setup shown in Figure 4. DEs for various spatial frequencies were measured. The modulation transfer function of the device in Figure 5 a shows the resolution to be $250 \mathrm{lp} \mathrm{mm}^{-1}$. A $405 \mathrm{~nm}$ laser is used for writing images throughout the experiment. We limit the laser power to $5 \mathrm{~mW}$ so that the image written on the OASLM can disappear within one second, as an increase in the laser power results in increased photocurrent, resulting in a longer decay time once the laser is switched off, thereby retaining the image for hours. It is

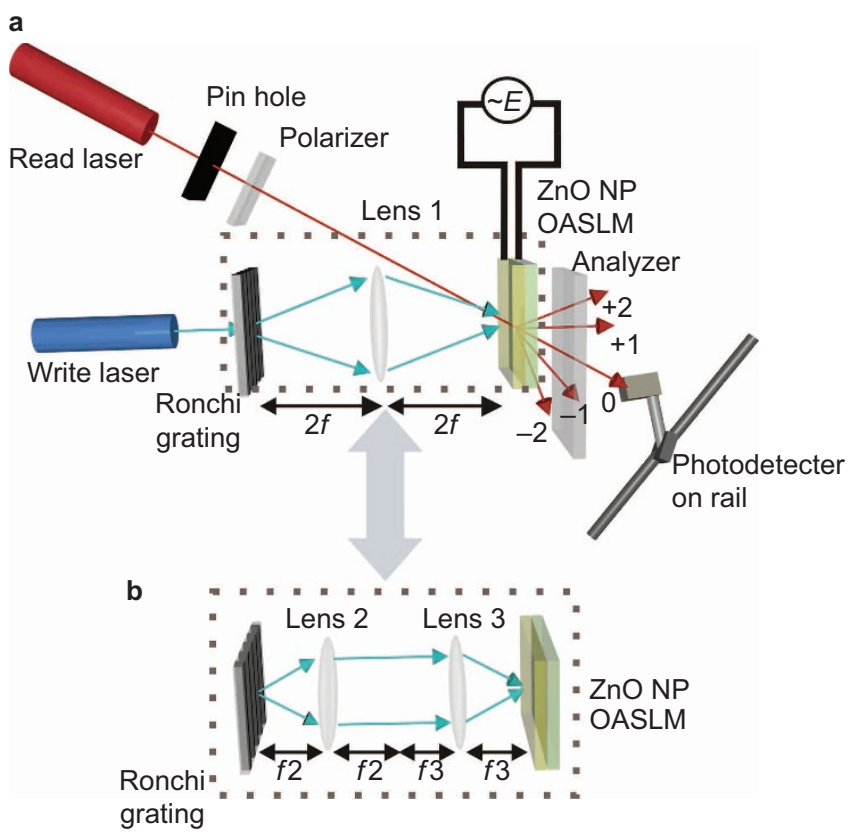

Figure 4 (a) Experimental setup for DE measurement and holographic imaging. (b) Two lens de-magnification optics. DE, diffraction efficiency; NP, nanoparticle; OASLM, optically addressed spatial light modulator.

observed that a low-resolution image is retained for longer. As the resolution of an image increases, the image retaining time decreases.

The DE can be increased by increasing the power of the write light at the expense of a lower image refresh time. As shown in Figure 4a, the imaging lens (achromatic doublet lens) is placed between a Ronchi diffraction grating and the OASLM at a distance of twice the focal length from either of them. Several Ronchi gratings with spatial fre-

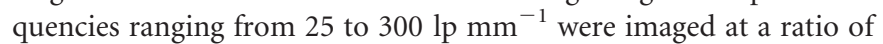
$1: 1$ onto the OASLM. For a read operation, a $635 \mathrm{~nm}$ laser of $2 \mathrm{~mW}$ is used. The polarizer and analyzer were placed orthogonal to each other after the read laser and OASLM. Higher spatial resolutions (355$825 \mathrm{lp} \mathrm{mm}^{-1}$ ) are created and imaged by de-magnifying the Ronchi grating using a two lens system. As shown in Figure $4 \mathrm{~b}$, the focal length of lens $2(f 2)$ is chosen to be larger than that of lens $3(f 3)$. When the Ronchi grating is placed at a distance $f 2$ before lens 2 and the distance between lens 2 and lens 3 is $(f 2+f 3)$, the image formed at distance $f 3$ from lens 3 will be de-magnified by a factor of $f 3 / f 2$.

The OASLM is operated in the phase modulation mode. The director of the NLC is pre-aligned in a direction parallel to the polarizer. The conductivity of the $\mathrm{ZnO}$ NP film in the region illuminated by the write light increases significantly. This prompts NLC molecules in the bright region to rotate relative to those in the dark regions, effectively transferring the image from the photosensor layer to the LC layer. At the same time, using a polarized read beam passing through the LC layer and the analyzer, the transferred image of the grating can be verified by measuring the angle between the zero- and first-order diffraction of the read light using Equation (3):

$$
a=\frac{m \lambda}{\sin \theta_{m}}
$$

where $a$ is the period of the imaged grating, $m$ is the order of diffraction and $\theta$ is the diffraction angle for the $m$ th order. The measurement of the angle between the zeroth- and first-order diffraction as the read light passes through the OASLM is presented in Figure $5 \mathrm{~b}$. 

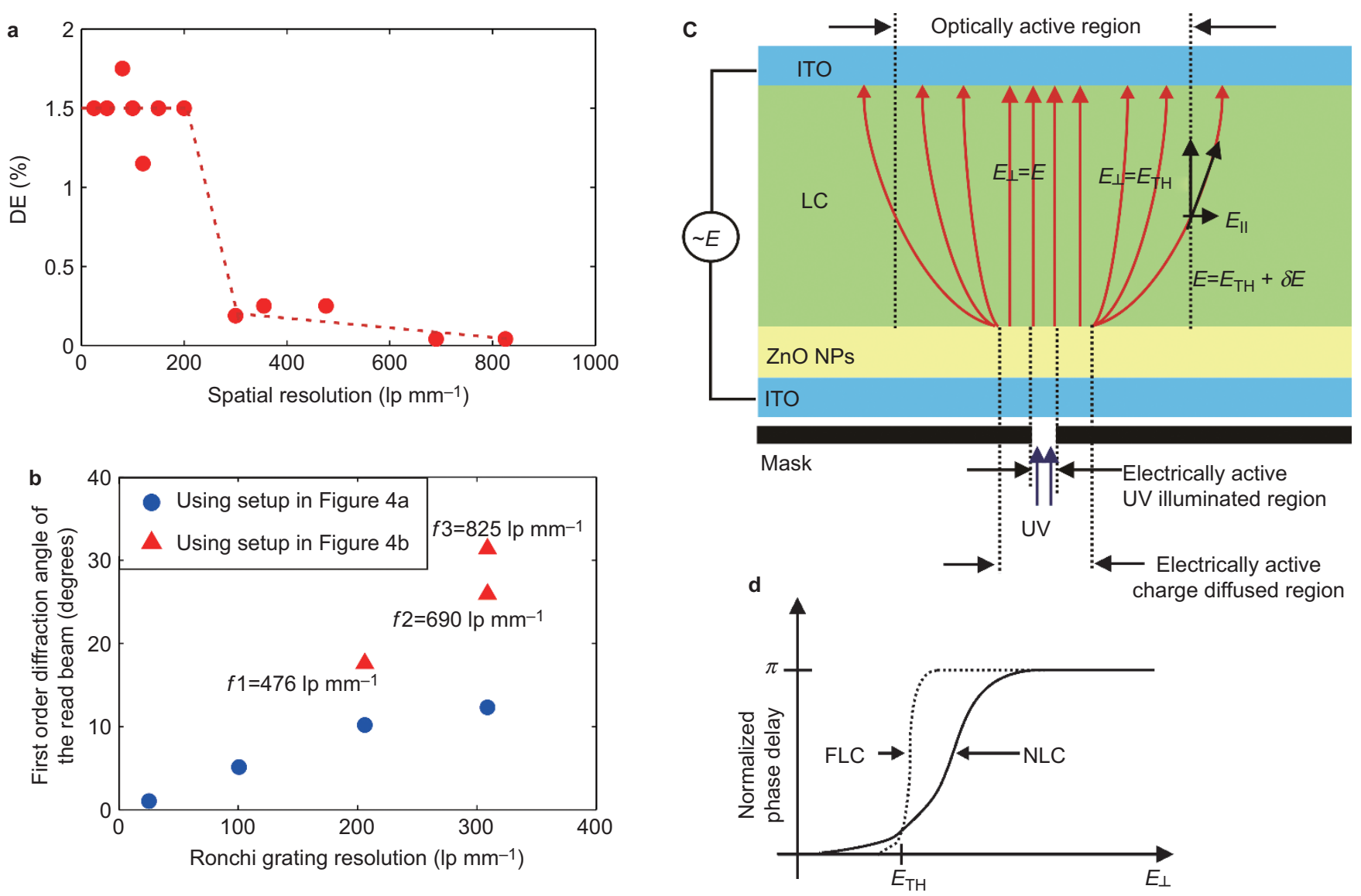

Figure 5 (a) Measured modulation transfer function. (b) The first order diffraction angle of the read beam vs. Ronchi grating resolution. Spatial frequencies $f 1, f 2$ and $f 3$ are produced using the setup in Figure 4b. (c) Electrically and optically active region in the OASLM. (d) Effect of the electric field on the LC. FLC, ferroelectric liquid crystal; ITO, indium tin oxide; LC, liquid crystal; NLC, nematic liquid crystal; NP, nanoparticle; OASLM, optically addressed spatial light modulator; UV, ultraviolet.

The turn-on and turn-off times of the photoresponse in colloidal $\mathrm{ZnO} \mathrm{NP}$ films were shown to be approximately $0.1 \mathrm{~s}$ and 9-10 s, respectively, for the photocurrent to change by three orders of magnitude. ${ }^{23}$ Although the specific values of the response times depend on device geometry, fabrication conditions and light illumination intensity, our observations are consistent with previously presented results. When the driving frequency of the write light is higher than a few tens of hertz, the modulation of the read light in accordance with the write light is no longer visible. This is most likely because the write time is insufficient to produce the charge distribution needed for the realignment of the photosensor-LC interface layer ${ }^{29}$ as a result of the high defect density in the NPs. ${ }^{17}$ Further research is needed to increase the refresh rate of such a system. A $0.2 \mathrm{~Hz}$ alternating current (AC) square wave of $6 \mathrm{~V}$ amplitude was used to drive the OASLM. A selected grating was imaged onto the OASLM. The intensities of the zeroth order $\left(I_{0}\right)$ and the first order $\left(I_{1}\right)$ of diffraction were recorded and used to calculate the firstorder DE. The DE is the measure of the ability of a device to diffract the optical power into a designated direction and is given by

$$
\mathrm{DE}=\frac{I_{1}}{I_{0}} \times 100 \%
$$

where $I_{1}$ is measured after the analyzer and $I_{0}$ is measured without any write light in the same configuration. This DE is termed the cell DE in the literature. ${ }^{30,31}$ The light intensity is measured at the end of a positive driving pulse using a power meter. All measurements are carried out in a dark room, and the background noise is subtracted from the recorded reading.

The measured DE is maintained at a constant level of approximately $1.5 \%$ for the gratings with a recorded resolution of up to $200 \mathrm{lp} \mathrm{mm}^{-1}$, as shown in Figure 5a. Beyond $200 \mathrm{lp} \mathrm{mm}^{-1}$, the DE is observed to decrease sharply. The DE for each resolution can be optimized separately to achieve a higher value of $\sim 10 \%$, but we have intentionally used a lower value of $\sim 1 \%$, which is better for the comparison of the performance over a large range of spatial resolutions. The thickness of the NLC used in our device was $5.5 \mu \mathrm{m}$ to ensure a phase change $>\pi$ when switched, but it is yet to be optimized. If we consider the ideal case of a device with infinitely large electrodes, the electric field direction in the LC is perpendicular to the electrodes. When the ratio of the pixel size to the LC cell thickness of a device is less than 1, the electric field at the edge of the electrodes deviates away from the surface normal, creating the field called the 'fringing electric field', as shown in Figure $5 \mathrm{c}$ and $5 \mathrm{~d}$. The fringing electric field is one of the most important factors that determine the resolution of a device. When the ratio of the layer thickness to the pixel size is much greater than 1 , the area affected by the applied electrical voltage will be much larger than that of the pixel electrode itself. Hence, electric field profiles tend to interfere with neighboring pixels, resulting in the nonideal phase profile of a LC. ${ }^{32,33}$

The resolution of the image formed by the imaging lens is limited by diffraction and is given by 


$$
(\Delta l)_{\min }=\frac{1.22 f^{\prime} \lambda}{D}
$$

where $(\Delta l)_{\min }$ is the minimum resolvable distance, $f^{\prime}$ is the distance between the lens and the focal point, $\lambda$ is the wavelength of light and $D$ is the lens diameter. For $1: 1$ imaging using the setup in Figure $4 a$, the diffraction limit imposed by the lens is calculated to be $1.94 \mu \mathrm{m}$. For the de-magnification setup in Figure 4b, the diffraction limit is calculated to be $0.61 \mu \mathrm{m}$. We use achromatic doublet lenses for both setups to minimize the chromatic and spherical aberrations. However, at higher spatial resolutions, monochromatic aberrations affect the image to some extent, degrading the quality of the Ronchi grating/ image imaged onto the OASLM. A higher spatial resolution grating will diffract the write light into a wider angle, resulting in only a few low orders being focused by the imaging lens. This decreases the contrast of the image formed on the photosensor layer and contributes to the lower DE.

The holograms recorded on the OASLM are replayed using a $635 \mathrm{~nm}$ wavelength read laser. Figure 6a illustrates the image produced by a $2 \mu \mathrm{m}$ pixel size binary hologram recorded on glass and illuminated by a $405 \mathrm{~nm}$ laser. Figure $6 \mathrm{~b}$ is the replay image produced by that hologram as recorded on the OASLM with $1: 1$ imaging. Figure $6 c$ shows the replay image reproduced by the OASLM recorded with de-magnified $(1: 0.42)$ imaging at a pixel size of $0.84 \mu \mathrm{m}$, and Figure $6 \mathrm{~d}$ shows the same image with de-magnified $(1: 0.36)$ imaging at a pixel size of $0.72 \mu \mathrm{m}$. Figure 6e shows the image of a dragon produced by a $2 \mu \mathrm{m}$ pixel size binary hologram recorded on glass and illuminated by a $405 \mathrm{~nm}$ laser. Figure $6 \mathrm{f}$ shows the replay of that hologram once it is recorded onto the OASLM with $1: 1$ imaging. For the holograms shown in Figure 6, the intensity of the write light is $\sim 40 \mathrm{~mW} \mathrm{~cm}^{-2}$.

\section{CONCLUSIONS}

In summary, we have been able to fabricate updatable high-resolution OASLMs with photoresponsive layers based on $\mathrm{ZnO}$ NPs. The fabrication process itself is simple and can be done at low temperatures

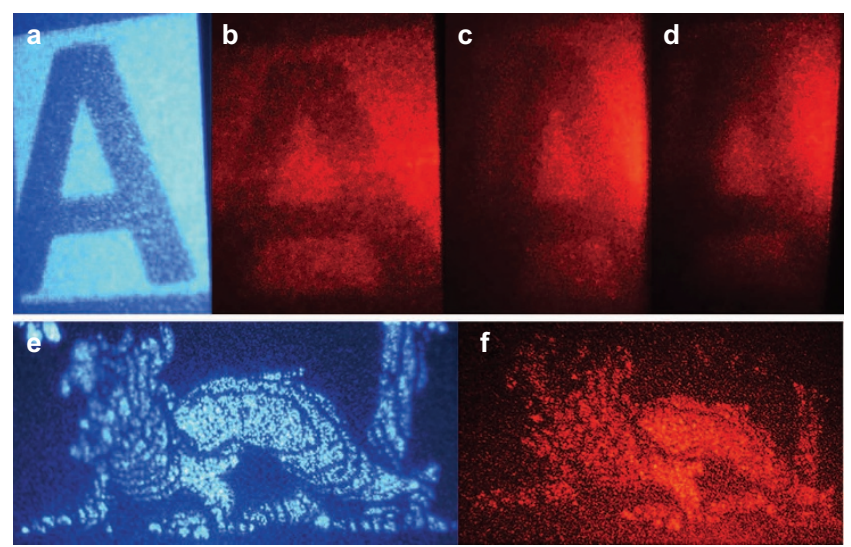

Figure 6 (a) Image produced by a $2 \mu \mathrm{m}$ pixel size hologram on glass as illuminated by a $405 \mathrm{~nm}$ laser; (b) replay of $1: 1$ image reproduced by the OASLM; (c) replay of de-magnified image $(1: 0.42)$ with a pixel size of $0.84 \mu \mathrm{m}$ reproduced by the OASLM; (d) replay of de-magnified image $(1: 0.36)$ with a pixel size of $0.72 \mu \mathrm{m}$ reproduced by the OASLM. (e) Image of a dragon generated by a $2 \mu \mathrm{m}$ pixel size hologram on glass as illuminated by a $405 \mathrm{~nm}$ laser; (f) replay of $1: 1$ image reproduced by the OASLM. OASLM, optically addressed spatial light modulator. $\left(\leqslant 180{ }^{\circ} \mathrm{C}\right)$. A resolution of up to $825 \mathrm{lp} \mathrm{mm}^{-1}$ has been achieved on an OASLM operated with a low AC voltage $(\leqslant 6 \mathrm{~V})$. We believe that this was made possible by two factors, a photoconductive film made of a thin layer of semiconductor NPs for minimum lateral charge and a NLC material with a reasonably sharp threshold voltage.

\section{ACKNOWLEDGEMENTS}

The authors would like to thank EPSRC for financial support through the Platform Grant for LC Photonics (EP/F00897X/1) and JS Chen for calculating the holograms used in Figure 6. They also would like to acknowledge the helps of I Medina, $\mathrm{K} \mathrm{Li}$ and N Collings at the initial stage of the experiments. PKS thanks the Schiff Foundation for the financial support of a Schiff Studentship.

1 Fukushima S, Kurokawa T, Ohno M. Real-time hologram construction and reconstruction using a high-resolution spatial light modulator. App/ Phys Lett 1991; 58: 787-789.

2 Wen L, Rice RA, Moddel G, Pagano-Stauffer LA, Handschy MA. Hydrogenated amorphous-silicon photosensor for optically addressed high-speed spatial light modulator. IEEE Trans Electron Dev 1989; 36: 2959-2964.

3 Efron U, Grinberg J, Braatz PO, Little MJ, Reif PG et al. The silicon liquid crystal light valve. J App/ Phys 1985; 57: 1356-1368.

4 Armitage D, Anderson W, Karr TJ. High-speed spatial light modulator. IEEE J Quantum Electron 1985; 21: 1241-1248.

5 Collings N, Pourzand AR, Vladimirov FL, Pletneva NI, Chaika AN. Pixelated liquidcrystal light valve for neural network application. App/ Opt 1999; 38: 6184-6189.

6 Kirzhner MG, Klebanov M, Lyubin V, Collings N, Abdulhalim I. Liquid crystal highresolution optically addressed spatial light modulator using a nanodimensional chalcogenide photosensor. Opt Lett 2014; 39: 2048-2051.

7 Collings N, Wilkinson TD, Jeziorska A, Davey AB, Movaghar B et al. Charge-injecting layers for liquid crystal light valves. Proc SPIE 2004; 5464: 421-427.

8 Wick DV, Martinez T, Wood MV, Wilkes JM, Gruneisen MT et al. Deformed-helix ferroelectric liquid-crystal spatial light modulator that demonstrates high diffraction efficiency and 370-line pairs/mm resolution. App/ Opt 1999; 38: 3798-3803.

9 White DL, Feldman M. Liquid-crystal light valves. Electron Lett 1970; 6: 837-839.

10 Aubourg P, Huignard JP, Hareng M, Mullen RA. Liquid crystal light valve using bulk monocrystalline $\mathrm{Bi}_{12} \mathrm{SiO}_{20}$ as the photoconductive material. Appl Opt 1982; 21 3706-3712.

11 Lynn B, Blanche PA, Peyghambarian N. Photorefractive polymers for holography. J Polym Sci Part B Polym Phys 2014; 52: 193-231.

12 Bartkiewicz S, Matczyszyn K, Miniewicz A, Kajzar F. High gain of light in photoconducting polymer-nematic liquid crystal hybrid structures. Opt Commun 2001; 187: 257-261.

13 Yao F, Pei Y, Zhang Y, Zhang J, Hou C et al. High-resolution photorefractive gratings in nematic liquid crystals sandwiched with photoconductive polymer film. Appl Phys $B$ 2008; 92: 573-576.

14 Wang L, Moddel G. Effects of charge spreading on resolution of optically addressed spatial light modulators. Opt Lett 1994; 19: 2033-2035.

15 Wang L, Moddel G. Resolution limits from charge transport in optically addressed spatial light modulators. J Appl Phys 1995; 78: 6923-6935.

16 Konstantatos G, Howard I, Fischer A, Hoogland S, Clifford J et al. Ultrasensitive solution-cast quantum dot photodetectors. Nature 2006; 442: 180-183.

17 Efros AL, Rosen M. The electronic structure of semiconductor nanocrystals. Annu Rev Mater Sci 2000; 30: 475-521.

18 Slinger C, Cameron C, Stanley M. Computer-generated holography as a generic display technology. Computer 2005; 38: 46-53.

19 Özgür Ü, Alivov YI, Liu C, Teke A, Reshchikov MA et al. A comprehensive review of ZnO materials and devices. J Appl Phys 2005; 98: 041301.

20 Carrey J, Carrère H, Kahn ML, Chaudret B, Marie X et al. Photoconductivity of selfassembled $\mathrm{ZnO}$ nanoparticles synthesized by organometallic chemistry. Semicond Sci Technol 2008; 23: 025003.

21 Janotti A, van de Walle CG. Fundamentals of zinc oxide as a semiconductor. Rep Prog Phys 2009; 72: 126501.

$22 \mathrm{Li} \mathrm{QH}$, Gao T, Wang YG, Wang TH. Adsorption and desorption of oxygen probed from ZnO nanowire films by photocurrent measurements. Appl Phys Lett 2005; 86: 123117.

23 Jin Y, Wang J, Sun B, Blakesley JC, Greenham NC. Solution-processed ultraviolet photodetectors based on colloidal ZnO nanoparticles. Nano Lett 2008; 8: 1649 1653.

24 Melnick DA. Zinc Oxide photoconduction, an oxygen adsorption process. J Chem Phys 1957; 26: 1136-1146.

25 van de Lagemaat J, Frank AJ. Nonthermalized electron transport in dye-sensitized nanocrystalline $\mathrm{TiO}_{2}$ films: transient photocurrent and random-walk modeling studies. J Phys Chem B 2001; 105: 11194-11205. 
26 Tiwana $\mathrm{P}$, Docampo $\mathrm{P}$, Johnston MB, Snaith HJ, Herz LM. Electron mobility and injection dynamics in mesoporous $\mathrm{ZnO}, \mathrm{SnO}_{2}$, and $\mathrm{TiO}_{2}$ films used in dyesensitized solar cells. ACS Nano 2011; 5: 5158-5166.

27 Koch U, Fojtik A, Weller H, Henglein A. Photochemistry of semiconductor colloids. Preparation of extremely small ZnO particles, fluorescence phenomena and size quantization effects. Chem Phys Lett 1985; 122: 507-510.

28 Bahnemann DW, Kormann C, Hoffmann MR. Preparation and characterization of quantum size zinc oxide: a detailed spectroscopic study. J Phys Chem 1987; 91 3789-3798.

29 Kaczmarek M, Dyadyusha A, Slussarenko S, Khoo IC. The role of surface charge field in two-beam coupling in liquid crystal cells with photoconducting polymer layers. J Appl Phys 2004: 96: 2616-2623.

30 Coomber SD, Cameron CD, Hughes JR, Sheerin DT, Slinger CW et al. Optically addressed spatial light modulators for replaying computer-generated holograms. Proc SPIE 2001; 4457: 9-19.
31 Mias S, Manolis IG, Collings N, Wilkinson TD, Crossland WA. Phase-modulating bistable optically addressed spatial light modulators using wide-switching-angle ferroelectric liquid crystal layer. Opt Eng 2005; 44: 014003.

32 Wang X, Wang B, McManamon PF, Pouch JJ, Miranda FA et al. Spatial resolution limitation of liquid crystal spatial light modulator. Proc SPIE 2004; 5553: 46-57.

33 Apter B, Efron U, Bahat-Treidel E. On the fringing-field effect in liquid-crystal beamsteering devices. App/ Opt 2004; 43: 11-19.

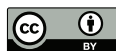

This work is licensed under a Creative Commons Attribution 3.0 Unported License. The images or other third party material in this article are included in the article's Creative Commons license, unless indicated otherwise in the credit line; if the material is not included under the Creative Commons license, users will need to obtain permission from the license holder to reproduce the material. To view a copy of this license, visit http://creativecommons.org/licenses/by/3.0/ 